\title{
Estabilidad del nivel del agua en un tanque con variaciones generadas por la demanda del servicio mediante modelos matemáticos
}

\section{Stability of the water level in a tank with variations generated by the demand for the service through mathematical models}

\author{
Nilthon Arce $\mathrm{F}^{1}$ (i)
}

\section{RESUMEN}

El objetivo de la investigación fue mantener estable el nivel del agua en un tanque con variaciones generadas por la demanda del servicio. Se emplearon dos modelos matemáticos que permitieron estudiar la estabilidad, el modelo no lineal representado por una ecuación diferencial y el modelo lineal por una función de transferencia. Se utilizó el software Matlab/Simulink mediante un método numérico de Runge-Kutta para modelar y simular el proceso. Con las condiciones iniciales del $90 \%$ en la apertura de la válvula de entrada y del $50 \%$ en la apertura de la válvula de salida, la solución de ambos modelos alcanzó un nivel estacionario de $\mathrm{h}=1,8 \mathrm{~m}$. Debido a la demanda del servicio, la válvula de entrada se reajustó en $+5 \%$, alcanzando la solución del sistema un nivel estacionario de $\mathrm{h}=2,005 \mathrm{~m}$ para el modelo no lineal y h=1,999 m para el modelo lineal. Finalmente, la válvula de salida se reajustó en $+3 \%$, alcanzando la solución del sistema un nivel estacionario de $\mathrm{h}=1.785 \mathrm{~m}$ para el modelo no lineal y $\mathrm{h}=1.784 \mathrm{~m}$ para el modelo lineal. Los modelos matemáticos empleados, permitieron estimar en menor tiempo con resultados esperados el nivel del agua, logrando así identificar la estabilidad del sistema.

Palabras clave: Modelo matemático, sistema dinámico, demanda, estabilidad, software.

\begin{abstract}
The objective of the research was to keep the water level in a tank stable with variations generated by the demand for the service. Two mathematical models were used that allowed studying the stability, the nonlinear model represented by a differential equation and the linear model by a transfer function. Matlab/Simulink scientific software was used by means of a Runge-Kutta numerical method to model and simulate the process. With the initial conditions of $90 \%$ at the inlet valve opening and $50 \%$ at the outlet valve opening, the solution of both models reached a stationary level of $h=1.8 \mathrm{~m}$. Due to service demand, the inlet valve was readjusted by $+5 \%$, with the system solution reaching a stationary level of $\mathrm{h}=2,005 \mathrm{~m}$ for the non-linear model and $\mathrm{h}=1,999 \mathrm{~m}$ for the linear model. Finally, the output valve was readjusted by $+3 \%$, with the system solution reaching a stationary level of $h=1,785 \mathrm{~m}$ for the non-linear model and $\mathrm{h}=1,784 \mathrm{~m}$ for the linear model. The mathematical models used, allowed to estimate in less time with expected results the water level, achieving this way to identify the stability of the system.
\end{abstract}

Keywords: Mathematical model, dynamic system, demand, stability, software.

DOI: https://doi.org/10.37787/pakamuros-unj.v8i4.152

Recibido: 27/08/2020. Aceptado: 01/11/2020

* Autor para correspondencia

1. Universidad Nacional de Jaén, Jaén, Perú. Email: nilthon_arce@unj.edu.pe 


\section{INTRODUCCIÓN}

Simular y a su vez modelar sistemas reales mediante ecuaciones matemáticas es una de las herramientas más aplicadas en la Ingeniería, hoy en día se abre un abanico de programas de simulación, que cumplen tales exigencias, talvez unos más estilizados y desarrollados que otros, sin embargo todos son útiles (Escalante \& Villacreses, 2015).

Según (Ogata, 1987), debido a su frecuencia en la industria, los circuitos hidráulicos y los sistemas hidráulicos constituyen una parte necesaria en la educación de un ingeniero. Muchos de los sistemas hidráulicos son no lineales. Sin embargo, algunas veces es posible linealizar sistemas no lineales de modo que se reduzca su complejidad y se obtengan soluciones que sean suficientemente exactas para muchos propósitos (Roa et al., 2017, p. 78).

El control de nivel en tanques y el flujo entre ellos es un problema que se presenta en una gran variedad de aplicaciones como en la industria petrolera, fabricación de alimentos y en las plantas potabilizadoras de agua, en donde se requiere el almacenamiento y la transferencia de productos entre tanques y hacia los consumidores, aun cuando se realicen mezclas en los tanques es necesario el control del nivel y regulación del flujo entre tanques. Los tanques de almacenamiento de agua sirven esencialmente como reservorios. Sin embargo, en el sistema de suministro de agua potable ellos equilibran los picos de la demanda de agua y mantienen presiones adecuadas en las redes de distribución. Al equilibrar la demanda evitan que las plantas de tratamiento de agua potable produzcan de forma instantánea lo que se necesita para el consumo en la hora pico, permitiendo que este tipo de plantas produzcan, la mayoría del tiempo, un caudal de potabilización constante e inferior al caudal pico, que reduce los requerimientos en instalaciones, equipos y materiales. De igual forma, controlan la presión de todos los conductos conectados a ellos. Se considera, además, que a mayor volumen en el depósito, más estable será la presión en el sistema de distribución, pues permiten un mayor control sobre la aparición de fenómenos transitorios (Inga, 2019).

Se tiene un tanque con área de base $A$ y altura máxima $H_{\max }$, el nivel del líquido en el tanque está controlado por dos válvulas, la válvula de entrada ubicada en la parte superior y la de salida en la parte inferior, las aperturas de las válvulas constituyen las variables de entrada del sistema, estas aperturas se regularán con cero cuando estén totalmente cerradas y con uno cuando estén totalmente abiertas, luego el nivel del líquido $(h)$ constituye la única variable de salida del sistema. 


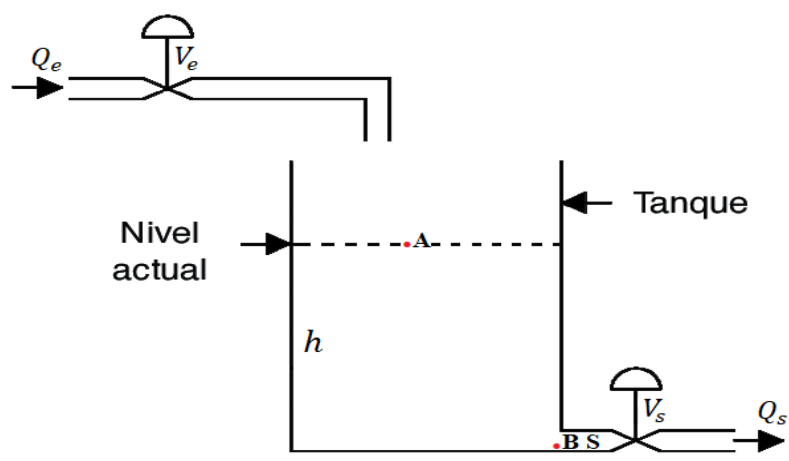

Figura 1. Sistema de control de un tanque operado por válvulas

Donde $h$ : altura del nivel del agua, $V_{e}$ : válvula de entrada, $Q_{e}$ : caudal de entrada regulada por $V_{e}, V_{s}$ : válvula de salida, $Q_{s}$ : caudal de salida regulada por $V_{s}$, S: sección transversal.

Para linealizar el modelo no lineal del problema planteado se utilizó la expansión de la serie de Taylor para funciones de dos variables hasta la segunda derivada o segundo orden (Rosa, 2013, p. 25).

En el presente trabajo se empleó dos modelos matemáticos que permitieron estimar la estabilidad del nivel del agua en un tanque a pesar de las variaciones generadas por la demanda del servicio, el modelo no lineal se representó a través de una ecuación diferencial ordinaria de primer orden, y el modelo lineal por una función de transferencia con un valor al nivel que garantizó el estado de equilibrio del tanque.

\section{MATERIALES Y MÉTODOS}

\section{Planteamiento del problema}

El tanque rectangular para almacenamiento de agua que se muestra en la Figura 2 tiene las características propias del tanque de la Figura 1, estimar el nivel del agua $(h)$ en menor tiempo con resultados esperados permitió identificar la estabilidad del sistema.

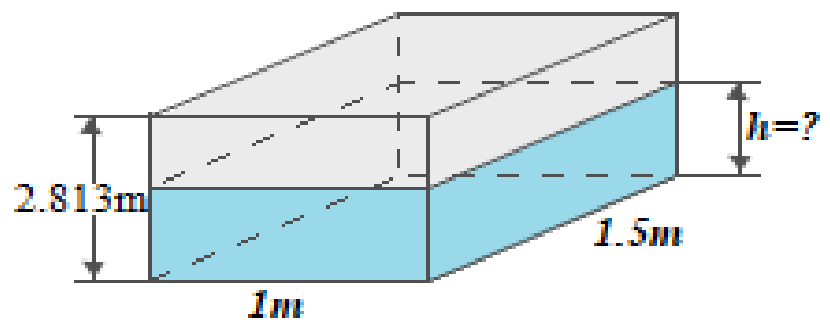

Figura 2. Tanque rectangular almacenamiento de agua

\section{Modelos matemáticos del problema planteado}

De la Figura 1, teniendo en cuenta que el nivel en el que se encuentra el orificio es el nivel de referencia, se observa que las presiones en la parte superior del tanque $\left(P_{1}\right)$ y en la salida del orificio $\left(P_{2}\right)$ son iguales a la presión atmosférica. Aplicando la ecuación de Bernoulli entre los puntos $A$ y $B$, obtenemos 
$P_{1}+\frac{1}{2} \rho v_{1}^{2}+\rho g h=P_{2}+\frac{1}{2} \rho v^{2}+\rho g h_{1}$. En el lado izquierdo de la ecuación, el segundo término puede igualarse a cero porque la velocidad del líquido en la parte superior del tanque (es decir, la velocidad con la que disminuye el nivel) puede ser despreciada. En el lado derecho de la ecuación, el tercer término también es igual a cero porque el nivel de referencia se encuentra a la altura del agujero. Recordando que $P_{1}=P_{2}=$ Patm la ecuación queda $P a t m+\rho g h=P a t m+\frac{1}{2} \rho v^{2}$. Luego, el valor de la velocidad del fluido a la salida del tanque es $v=\sqrt{2 g h}($ Gosmann, 2002, p. 77).

Se sabe que el caudal de salida está dado por el producto entre la velocidad del fluido y el área transversal por la cual circula (Fernández, 2015)

$$
Q=A V=A \sqrt{2 g h}
$$

Considerando que el caudal de entrada $Q_{e}$ es proporcional a la apertura de la válvula de entrada $\left(V_{e}\right)$ se tiene que: $Q_{e}=k_{1} a_{1}$, donde $k_{1}$ es constante y $a_{1}$ representa la apertura de la válvula de entrada $\left(V_{e}\right)$. Es importante mencionar que esto aplica a un sistema con suministro constante. Para calcular el caudal de salida $Q_{s}$ hacemos una aproximación lineal entre $Q_{s}$ y la apertura de la válvula de salida $\left(V_{S}\right)$, considerando la diferencia de presiones en $V_{S}$ constante y el fluido que hay dentro del tanque se tiene que: $Q_{s}=k_{2} a_{2} \sqrt{2 g h}$; donde $k_{2}$ es constante, $a_{2}$ representa la apertura de la válvula de salida $\left(V_{s}\right)$ (Castaño, s. f.)

El líquido acumulado en el interior, resulta la diferencia entre el flujo entrante y el flujo saliente del tanque. Entonces, debe satisfacerse la ecuación (Loyarte \& Clementi, 2011, p. 966).

$$
A \frac{d h}{d t}=Q_{e}-Q_{s}
$$

Es decir, el área de la base del tanque por la variación del nivel del agua con respecto al tiempo es igual a lo que entra $\left(Q_{e}\right)$ menos lo que sale $\left(Q_{s}\right)$.

\section{Modelo matemático no lineal}

Remplazando $Q_{e}, Q_{s}$ en (2) se obtuvo la ecuación diferencial ordinaria de primer grado que representa al modelo no lineal del sistema de control de un tanque operado por válvulas

$$
A \frac{d h}{d t}=k_{1} a_{1}-k_{2} a_{2} \sqrt{2 g h}
$$

\section{Modelo matemático lineal}

Para linealizar el modelo matemático anterior, se calculó un punto de equilibrio en el cual la altura $h$ se mantiene quieta, es decir no hubo variación de $h$, entonces $Q_{e}=Q_{s}$ indica el punto de equilibrio del 
sistema (Roa et al., 2017, p. 88). Así de la ecuación $k_{1} a_{1}=k_{2} a_{2} \sqrt{2 g \bar{h}}$ despejando $\bar{h}$ se obtiene la ecuación (4):

$$
\bar{h}=H_{o}=\left[\frac{k_{1} a_{1 E}}{k_{2} a_{2 E} \sqrt{2 g}}\right]^{2}
$$

Donde $\bar{h}$ : nivel de agua del tanque en estado de equilibrio, $a_{1 E}$ : apertura de $V_{e}$ en el punto de equilibrio, $a_{2 E}$ : apertura de $V_{S}$ en el punto de equilibrio.

Se empleó la serie de Taylor para linealizar el tanque en $\bar{h}$, considerando $a_{2}$ que es la apertura de $V_{s}$ como una perturbación, es decir $a_{2}$ constante (fija) y variando $a_{1}$ que es la apertura de $V_{e}$ (válvula de control) (Castaño, s. f.)

$$
\begin{gathered}
f\left(a_{1}, h\right)=A \frac{d h}{d t}=k_{1} a_{1}-k_{2} a_{2} \sqrt{2 g h} \\
f\left(a_{1}, h\right) \approx f\left(\overline{a_{1}}, \bar{h}\right)+\frac{d f}{d a_{1}}\left(\overline{a_{1}}, \bar{h}\right) \Delta a_{1}+\frac{d f}{d h}\left(\overline{a_{1}}, \bar{h}\right) \Delta h
\end{gathered}
$$

donde: $\Delta a_{1}=a_{1}-\overline{a_{1}} ; \Delta h=h-\bar{h}$.

Derivando en (5) y sustituyendo en (6) se tiene:

$$
\begin{gathered}
f\left(a_{1}, h\right) \approx f\left(\overline{a_{1}}, \bar{h}\right)+k_{1} \Delta a_{1}-\frac{k_{2} a_{2} \sqrt{2 g}}{2 \sqrt{\bar{h}}} \Delta h \\
f\left(a_{1}, h\right)-f\left(\overline{a_{1}}, \bar{h}\right) \approx k_{1} \Delta a_{1}-\frac{k_{2} a_{2} \sqrt{2 g}}{2 \sqrt{\bar{h}}} \Delta h \\
f\left(\Delta a_{1}, \Delta h\right) \approx k_{1} \Delta a_{1}-\frac{k_{2} a_{2} \sqrt{2 g}}{2 \sqrt{\bar{h}}} \Delta h \\
A \frac{d \Delta h}{d t} \approx k_{1} \Delta a_{1}-\frac{k_{2} a_{2} \sqrt{2 g}}{2 \sqrt{\bar{h}}} \Delta h
\end{gathered}
$$

El análisis no termina, hasta poder obtener la función de transferencia y saber a qué orden pertenece el sistema. Para poder obtenerla, aplicamos Laplace (Escalante \& Villacreses, 2015, p. 61)

$$
\mathcal{L}\left\{\frac{d \Delta h}{d t}\right\}=\operatorname{sL}\{\Delta h\}=\operatorname{sh}(s) \quad, \quad \mathcal{L}\left\{\Delta a_{1}\right\}=a_{1}(s)
$$

Aplicando esto a la ecuación (10) se consigue la función de transferencia, ecuación (11).

$$
\frac{h(s)}{a_{1}(s)}=\frac{k_{1}}{A s+\frac{k_{2} \overline{a_{2}} \sqrt{2 g}}{2 \sqrt{\bar{h}}}}=F(s)
$$


De manera análoga se empleó Taylor para linealizar el tanque en $\bar{h}$, considerando $a_{1}$ que es la apertura de $V_{e}$ como una perturbación, es decir $a_{1}$ constante (fija) y variando $a_{2}$ que es la apertura de $V_{s}$ (válvula de control) (Castaño, s. f.)

$$
A \frac{d \Delta h}{d t} \approx-k_{2} \sqrt{2 g \bar{h}} \Delta a_{2}-\frac{k_{2} \overline{a_{2}} \sqrt{2 g}}{2 \sqrt{\bar{h}}} \Delta h
$$

donde: $\Delta a_{2}=a_{2}-\overline{a_{2}}$.

Aplicando la transformada de Laplace a la aproximación lineal (12) se obtiene la función de transferencia

$$
\frac{h(s)}{a_{2}(s)}=-\frac{k_{2} \sqrt{2 g \bar{h}}}{A s+\frac{k_{2} \overline{a_{2}} \sqrt{2 g}}{2 \sqrt{\bar{h}}}}=G(s)
$$

Finalmente, la función de transferencia general que representa al modelo lineal del sistema de control de un tanque operado por válvulas, se expresa como la ecuación (14):

$$
J(s)=F(s)+G(s)=\frac{k_{1}}{A s+\frac{k_{2} \overline{a_{2}} \sqrt{2 g}}{2 \sqrt{\bar{h}}}}-\frac{k_{2} \sqrt{2 g \bar{h}}}{A s+\frac{k_{2} \overline{a_{2}} \sqrt{2 g}}{2 \sqrt{\bar{h}}}}
$$

\section{Simulación del problema planteado}

Se utilizó el software científico Matlab/Simulink mediante un método numérico de Runge-Kutta para modelar y simular el proceso. Los métodos de Runge-Kutta (RK) logran la exactitud del procedimiento de la serie de Taylor sin necesitar el cálculo de derivadas de orden superior. Existen muchas variantes, pero todas tienen la forma generalizada de la ecuación $y_{i+1}=y_{i}+\phi h$ (Chapra et al., 2007). Se consideró los siguientes parámetros: $A=1.5 \mathrm{~m}^{2}$ [área de la base del tanque], $H_{\max }=2.813 \mathrm{~m}$ [altura máxima del tanque], $k_{1}=0.05 \mathrm{~m}^{3} / \mathrm{s}$ [constante de $V_{e}$ ], $k_{2}=0.015 \mathrm{~m}^{3} / \mathrm{s}$ [constante de $V_{s}$ ], $a_{1 E}=0.9$ [apertura de $V_{e}$ ], $a_{2 E}=0.5$ [apertura de $V_{s}$ ], $g=10 \mathrm{~m} / \mathrm{s}^{2}$ [gravedad].

\section{RESULTADOS}

Nivel del agua en estado de equilibrio: $\bar{h}=H_{o}=\left[\frac{k_{1} a_{1 E}}{k_{2} a_{2 E} \sqrt{2 g}}\right]^{2}=1,8 \mathrm{~m}$ 
En la Figura 3, se observa la variación en la apertura de las válvulas del sistema, considerando $a_{2}=$ $a_{2 E}=0.5$ fija y variando $a_{1}$, luego considerando $a_{1}=a_{1 E}=0.9$ fija y variando $a_{2}$.
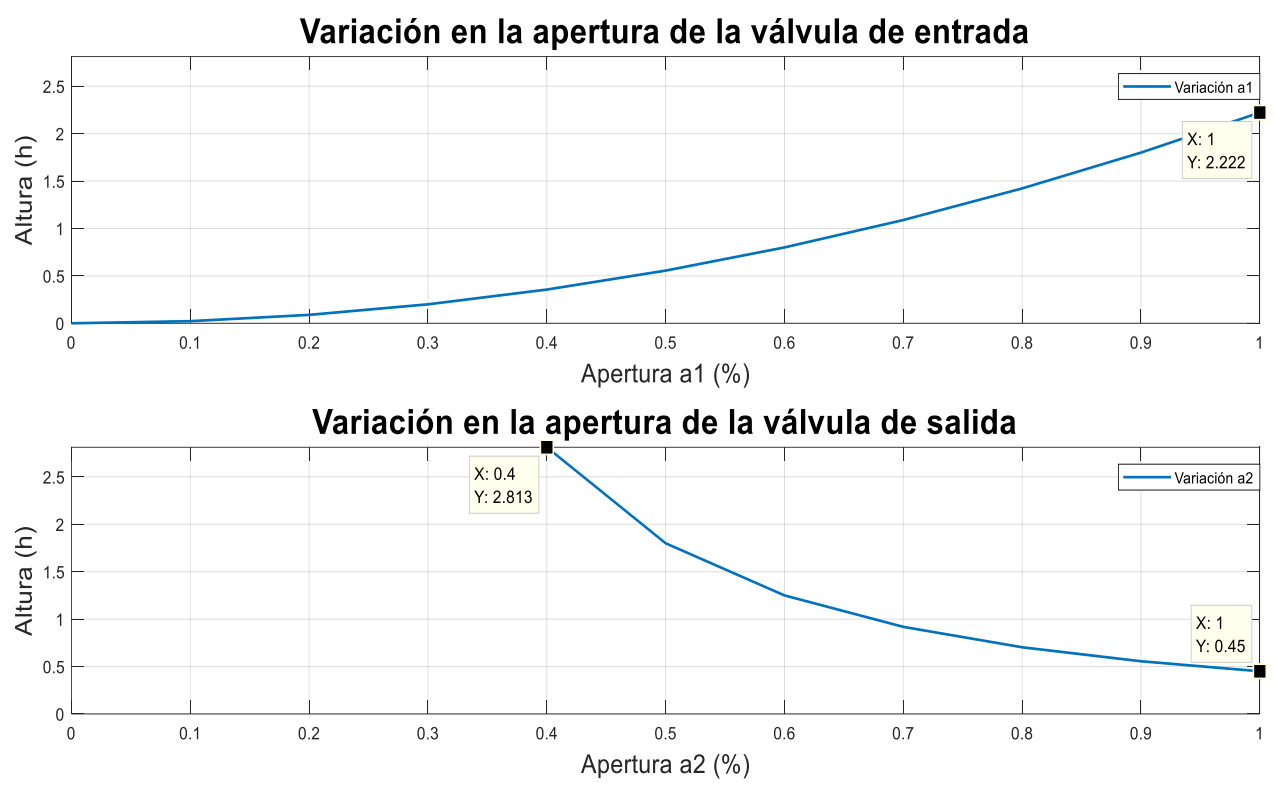

Figura 3. Variación en la apertura de las válvulas del sistema

En la Figura 4, se observa la solución del modelo no lineal del sistema, donde $a_{1 E}=90 \%$ (apertura de $V_{e}$ ) у $a_{2 E}=50 \%$ (apertura de $V_{s}$ ) son las variables de entrada, siendo el nivel de agua del tanque $(h)$ la única variable de salida, en la solución del modelo lineal del sistema, además de las variables anteriores se tiene otra variable de entrada que es el nivel de agua del tanque en estado de equilibrio $(H o=1,8 \mathrm{~m})$.
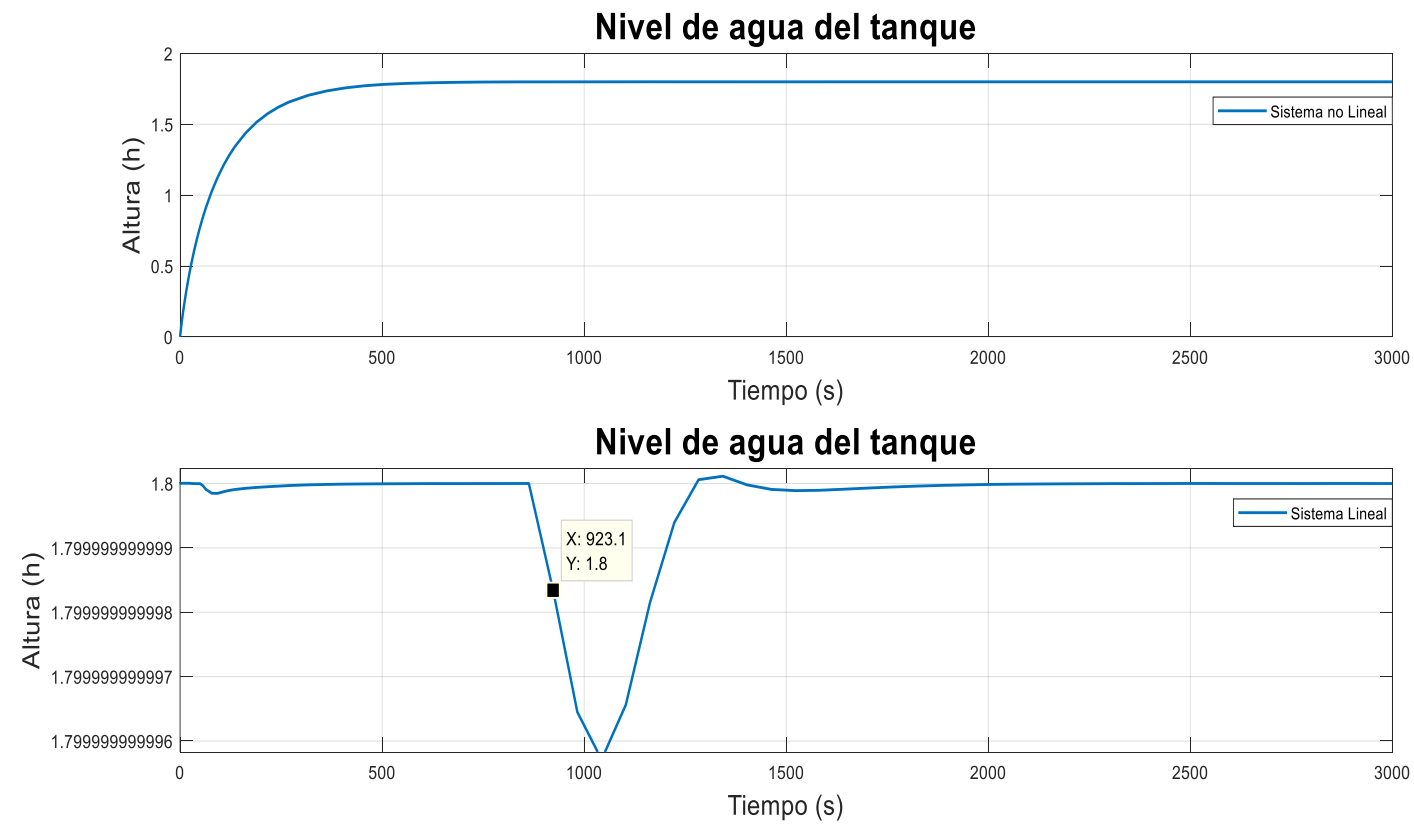

Figura 4. Solución del modelo no lineal y lineal del sistema de control de un tanque operado por válvulas 
En la Figura 5, se observa la solución del modelo no lineal junto a la solución del modelo lineal del sistema, en ella se verifica la similitud de estas soluciones en $H o=1.8 \mathrm{~m}$ (nivel de agua del tanque en estado de equilibrio).

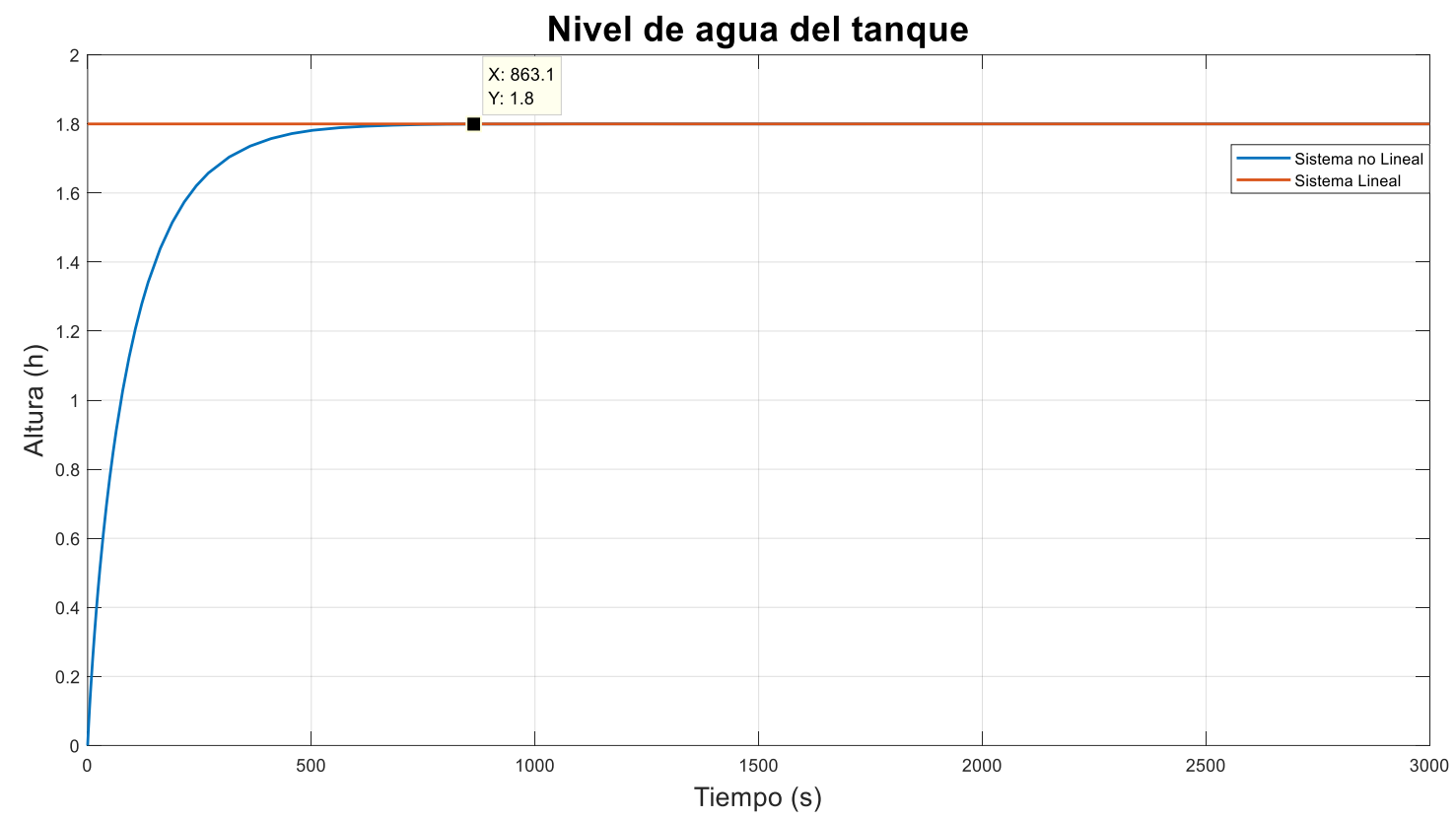

Figura 5. Solución modelo no lineal Vs solución modelo lineal

En la Figura 6, se observa el proceso de simulación de la solución del modelo no lineal y lineal del sistema de control de un tanque operado por válvulas, variando en determinados tiempos la apertura de la válvula de entrada $\left(a_{1 E}=90 \%\right)$ en $+5 \%$ y la apertura de la válvula de salida $\left(a_{2 E}=50 \%\right)$ en $+3 \%$.

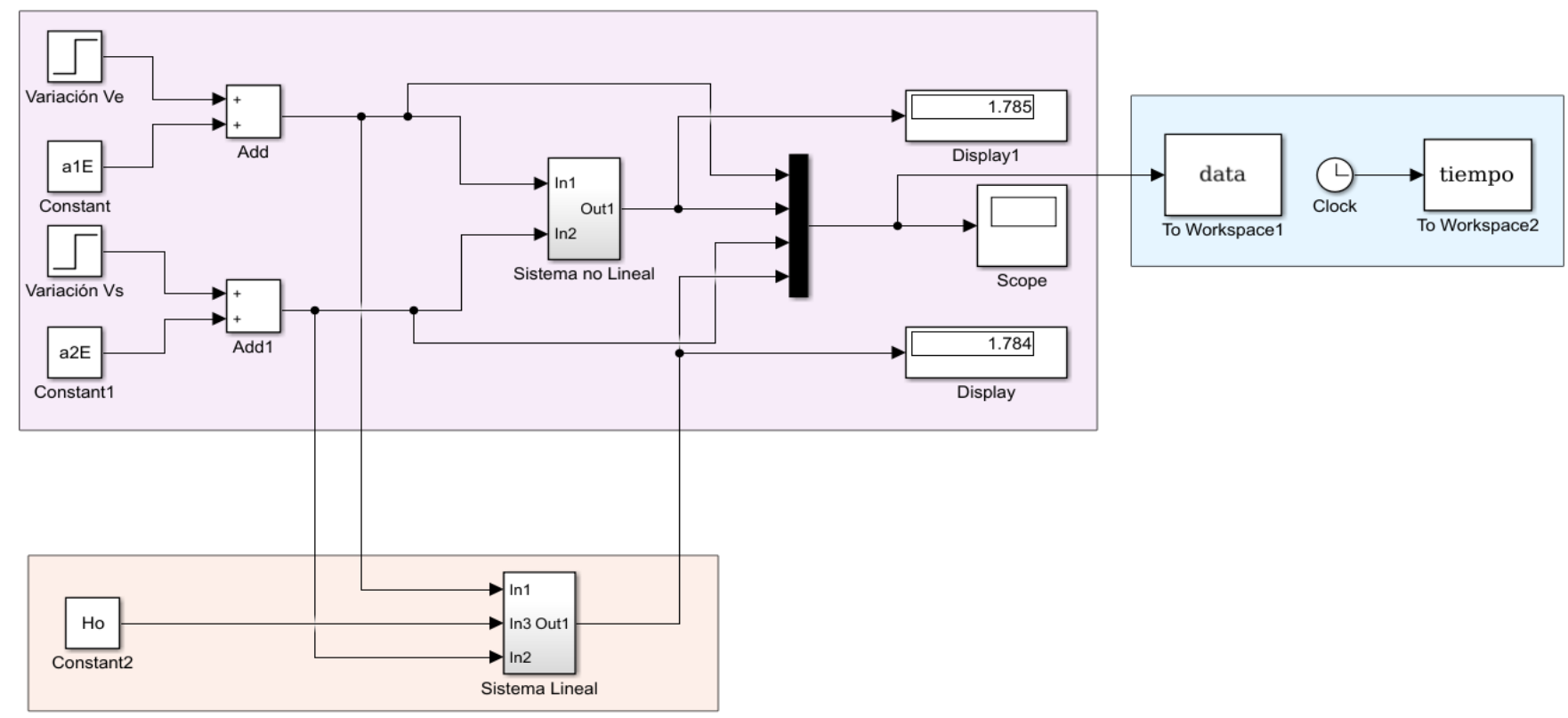

Figura 6. Proceso de simulación de la solución no lineal y lineal del sistema de control de un tanque operado por válvulas 
En la Figura 7, se observa la simulación de la solución del modelo no lineal junto a la simulación de la solución del modelo lineal del sistema de control de un tanque operado por válvulas, considerando una variación en la apertura de la válvula de entrada de $+5 \%$ y una variación en la apertura de la válvula de salida de $+3 \%$.
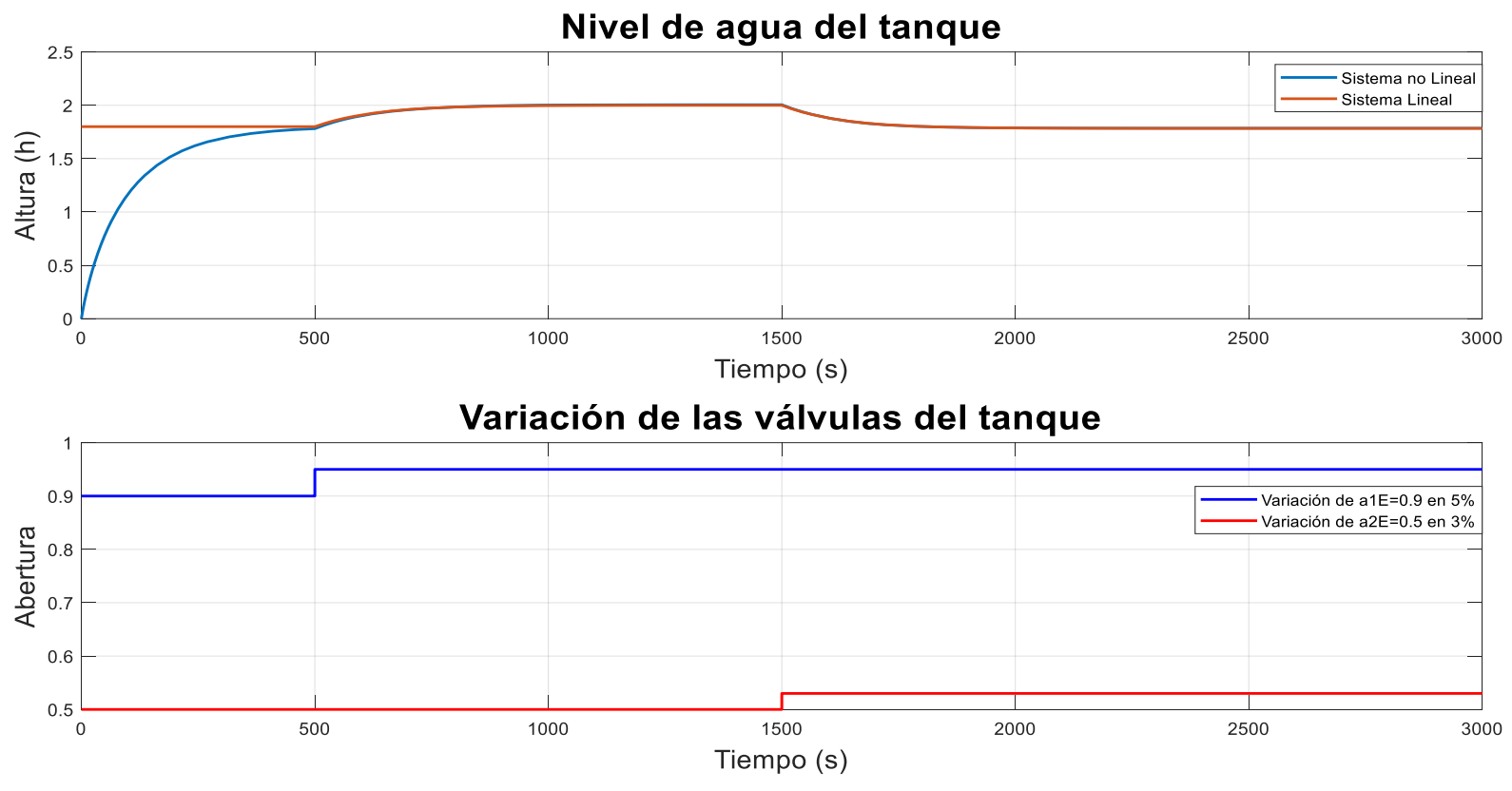

Figura 7. Simulación con ambos modelos del sistema variando la apertura de las válvulas

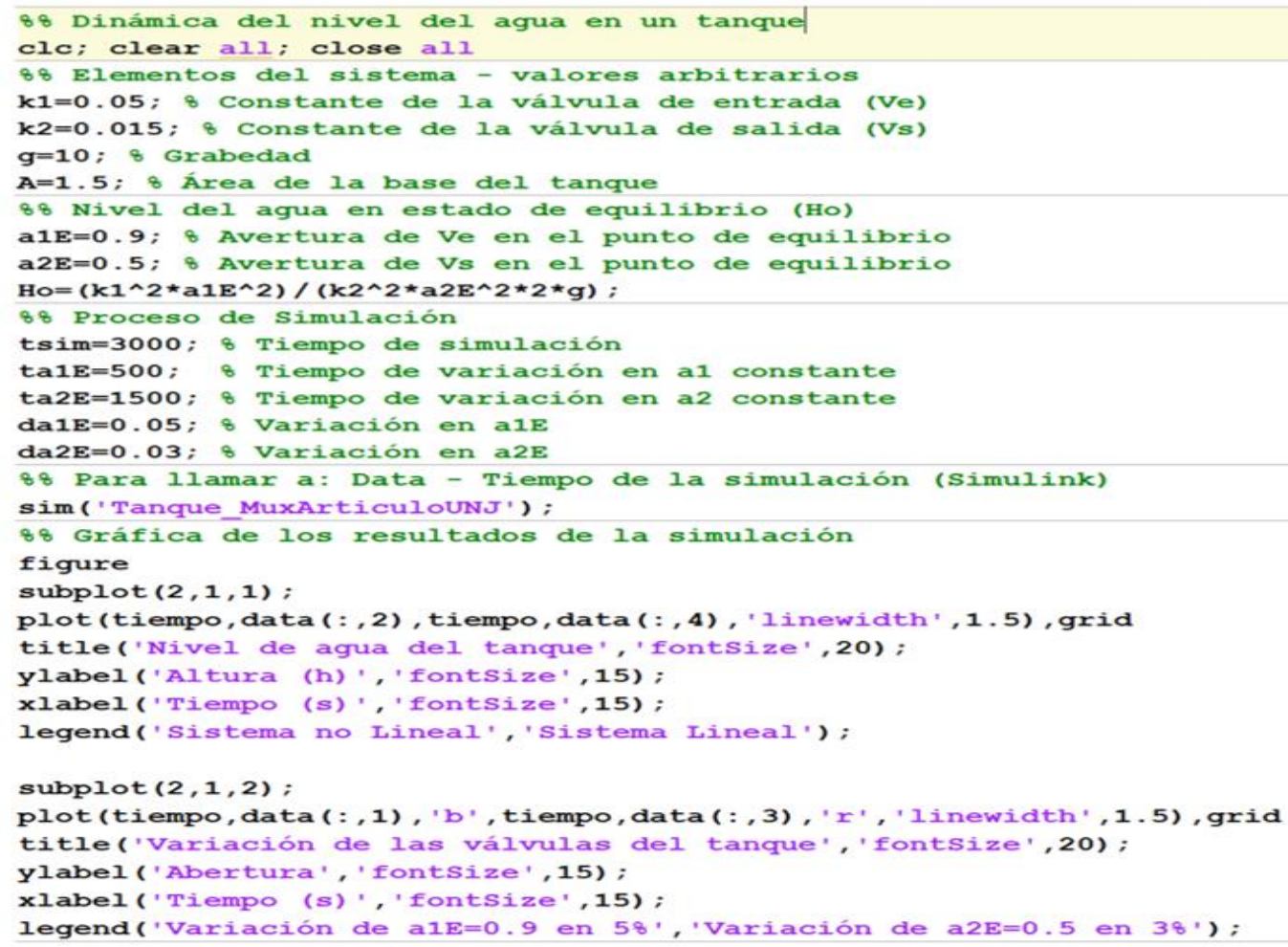

Figura 8. Código de simulación

La Tabla 1, muestra la data de simulación de la solución no lineal y lineal del sistema antes y después de los tiempos $(t=500 \mathrm{~s}, t=1500 \mathrm{~s})$, en el tiempo de simulación $t=500 \mathrm{~s}$ la apertura de la válvula de 
entrada varía en $+5 \%$, y en el tiempo de simulación $t=1500 \mathrm{~s}$ la apertura de la válvula de salida varía en $+3 \%$. Muestra también que ambos modelos del sistema siempre tienden a la misma solución.

Tabla 1. Data de simulación de la solución no lineal y lineal del sistema

\begin{tabular}{|c|c|c|c|c|}
\hline Tiempo & Abertura $V_{e}$ & Abertura $V_{s}$ & Sistema no lineal & Sistema lineal \\
\hline . & - & . & . & - \\
\hline . & . & . & . & . \\
\hline . & . & . & . & . \\
\hline 409,824650960868 & 0,900000000000000 & 0,500000000000000 & 1,75747747684275 & 1,79999999999999 \\
\hline 456,477764720835 & 0,900000000000000 & 0,500000000000000 & 1,77213818130053 & 1,79999999999999 \\
\hline 499,999999999996 & 0,900000000000000 & 0,500000000000000 & 1,78115638742591 & 1,80000000000000 \\
\hline 500 & 0,950000000000000 & 0,500000000000000 & 1,78115638742591 & 1,80000000000000 \\
\hline 500,000000000004 & 0,950000000000000 & 0,500000000000000 & 1,78115638742592 & 1,80000000000000 \\
\hline 520,995333964661 & 0,950000000000000 & 0,500000000000000 & 1,81500509711767 & 1,83049041923405 \\
\hline 541,990667929319 & 0,950000000000000 & 0,500000000000000 & 1,84397273832745 & 1,85644580326668 \\
\hline 562,986001893976 & 0,950000000000000 & 0,500000000000000 & 1,86854663373596 & 1,87844507089738 \\
\hline 583,981335858634 & 0,950000000000000 & 0,500000000000000 & 1,88935136290306 & 1,89707593490002 \\
\hline 623,418771472172 & 0,950000000000000 & 0,500000000000000 & 1,92052202850820 & 1,92565690968443 \\
\hline 662,856207085711 & 0,950000000000000 & 0,500000000000000 & 1,94316323102631 & 1,94668328020476 \\
\hline 702,293642699249 & 0,950000000000000 & 0,500000000000000 & 1,95957019227853 & 1,96185923734398 \\
\hline 741,731078312787 & 0,950000000000000 & 0,500000000000000 & 1,97154623170609 & 1,97272321176112 \\
\hline 801,731078312787 & 0,950000000000000 & 0,500000000000000 & 1,98423991362839 & 1,98342233766056 \\
\hline 861,731078312787 & 0,950000000000000 & 0,500000000000000 & 1,99281994493371 & 1,98981413782397 \\
\hline 921,731078312787 & 0,950000000000000 & 0,500000000000000 & 1,99848782743940 & 1,99374743710444 \\
\hline 981,731078312787 & 0,950000000000000 & 0,500000000000000 & 2,00176613357335 & 1,99619730791898 \\
\hline 1041,73107831279 & 0,950000000000000 & 0,500000000000000 & 2,00333636322612 & 1,99770336460359 \\
\hline 1101,73107831279 & 0,950000000000000 & 0,500000000000000 & 2,00400634385149 & 1,99861110027238 \\
\hline 1161,73107831279 & 0,950000000000000 & 0,500000000000000 & 2,00450374276113 & 1,99915464005594 \\
\hline 1221,73107831279 & 0,950000000000000 & 0,500000000000000 & 2,00502973891183 & 1,99948336208712 \\
\hline 1281,73107831279 & 0,950000000000000 & 0,500000000000000 & 2,00536853513507 & 1,99968720701874 \\
\hline 1341,73107831279 & 0,950000000000000 & 0,500000000000000 & 2,00550963805209 & 1,99981250559984 \\
\hline 1401,73107831279 & 0,950000000000000 & 0,500000000000000 & 2,00550498296447 & 1,99988828492016 \\
\hline 1461,73107831279 & 0,950000000000000 & 0,500000000000000 & 2,00543565705456 & 1,99993357286272 \\
\hline 1499,99999999999 & 0,950000000000000 & 0,500000000000000 & 2,00543611997226 & 1,99994931643139 \\
\hline 1500 & 0,950000000000000 & 0,530000000000000 & 2,00543611997226 & 1,99994931643139 \\
\hline 1500,00000000001 & 0,950000000000000 & 0,530000000000000 & 2,00543611997224 & 1,99994931643136 \\
\hline 1521,58024415085 & 0,950000000000000 & 0,530000000000000 & 1,96989587960243 & 1,96623106664248 \\
\hline 1543,16048830169 & 0,950000000000000 & 0,530000000000000 & 1,93985663223591 & 1,93764925520406 \\
\hline 1564,74073245254 & 0,950000000000000 & 0,530000000000000 & 1,91460488388685 & 1,91352971211998 \\
\hline 1604,27043507250 & 0,950000000000000 & 0,530000000000000 & 1,87710375805050 & 1,87751224642913 \\
\hline 1643,80013769247 & 0,950000000000000 & 0,530000000000000 & 1,84980070427301 & 1,85101594737561 \\
\hline 1683,32984031244 & 0,950000000000000 & 0,530000000000000 & 1,83037781733418 & 1,83190182076936 \\
\hline 1722,85954293241 & 0,950000000000000 & 0,530000000000000 & 1,81671857997299 & 1,81822906380656 \\
\hline 1782,85954293241 & 0,950000000000000 & 0,530000000000000 & 1,80366171092097 & 1,80480156219721 \\
\hline 1842,85954293241 & 0,950000000000000 & 0,530000000000000 & 1,79614568435290 & 1,79678116681992 \\
\hline 1902,85954293241 & 0,950000000000000 & 0,530000000000000 & 1,79166767918067 & 1,79184608225883 \\
\hline 1962,85954293241 & 0,950000000000000 & 0,530000000000000 & 1,78894209658654 & 1,78877203681724 \\
\hline 2022,85954293241 & 0,950000000000000 & 0,530000000000000 & 1,78729661470635 & 1,78688205625891 \\
\hline 2082,85954293241 & 0,950000000000000 & 0,530000000000000 & 1,78630868642302 & 1,78572651578762 \\
\hline 2142,85954293241 & 0,950000000000000 & 0,530000000000000 & 1,78572361719894 & 1,78502903354466 \\
\hline 2202,85954293241 & 0,950000000000000 & 0,530000000000000 & 1,78538430791614 & 1,78461218959267 \\
\hline 2262,85954293241 & 0,950000000000000 & 0,530000000000000 & 1,78519049946895 & 1,78436424295466 \\
\hline 2322,85954293241 & 0,950000000000000 & 0,530000000000000 & 1,78509111703464 & 1,78422893636395 \\
\hline$\cdot$ & • & • & $\cdot$ & $\cdot$ \\
\hline - & - & - & - & - \\
\hline - & - & - & - & - \\
\hline
\end{tabular}




\section{DISCUSIÓN}

Las aperturas $\mathrm{a}_{1}, \mathrm{a}_{2}$ de la válvula de entrada y salida respectivamente, se regulan con 0 cuando estén totalmente cerradas o 1 cuando estén totalmente abiertas. Para a_2E=50\% [apertura de Vs] fija con a1 apertura de $\mathrm{V}_{\mathrm{e}}$ variando, el nivel del agua es ascendente pero no alcanza la altura máxima del tanque [H_max=2,813m], tal como se observa en la primera gráfica de la Figura 3; para a_1E=90\% [apertura de $\mathrm{V}_{\mathrm{e}}$ ] fija con $\mathrm{a}_{2}$ apertura de $\mathrm{V}_{\mathrm{s}}$ variando, el nivel del agua desciende des de $\mathrm{H} \_\mathrm{max}=2,813 \mathrm{~m}$ hasta $\mathrm{h}=0,45 \mathrm{~m}$ tal como se observa en la Figura 3.

En la presente investigación se evaluaron tres casos que permiten estudiar la estabilidad del nivel del agua en un tanque con variaciones generadas por la demanda del servicio, considerando los modelos matemático no lineal y lineal del sistema obtenidos de marea detallada en la presenta investigación se logró resultados favorables para la misma, no logrados por Anaya et al. (2018) que concluyeron: disponiendo del modelo matemático que representa la dinámica de un equipo de dos tanques interconectados con las características planteadas en el presente trabajo, se logró el objetivo de realizar un análisis, basado en sus propiedades, sobre el comportamiento en el tiempo y el espacio de estado, obteniendo como resultado que a este modelo no se le encuentran puntos de equilibrio resolviendo por linealización o por el método numérico de Runge-Kutta.

Primero se ejecutó la simulación para a_1E=90\% y a_2E=50\%, se verifica en la Figura 4 que la solución de ambos modelos alcanzó un nivel estacionario de H_o=1,8m, que es justo el nivel del agua del tanque en estado de equilibrio considerado para la solución del modelo lineal, en la Figura 5 se confirma este resultado. En el tiempo de simulación $\mathrm{t}=500 \mathrm{~s}$ se manipuló la apertura a_1E=90\% en más 5\% aumentando el nivel del agua, en la Tabla 1 se verifica que a partir del tiempo de simulación t=1221,731s la solución de los modelos alcanzó un nivel estacionario de $\mathrm{h}=2,005 \mathrm{~m}$ para el modelo no lineal y h=1,999m para el modelo lineal, esto permite afirmar que los modelos del sistema tienden a la misma solución (e=0.006). Finalmente, en el tiempo de simulación $\mathrm{t}=1500 \mathrm{~s}$ se manipuló la apertura a_2E=50\% en más $3 \%$ disminuyendo el nivel del agua, en la Tabla 1 se verifica que a partir del tiempo de simulación $\mathrm{t}=2202,859 \mathrm{~s}$ la solución de los modelos alcanzó un nivel estacionario de $\mathrm{h}=1,785 \mathrm{~m}$ para el modelo no lineal y $\mathrm{h}=1,784 \mathrm{~m}$ para el modelo lineal, esto permite afirmar nuevamente que los modelos del sistema tienden a la misma solución (e=0.001); el Display - Display1 de la Figura 6 con la Figura 7 concluyen que ambos modelos tienden a la misma solución del sistema. Así coincido con los resultados obtenidos por Anzurez et al. (2008) que concluyeron: el ejemplo de aplicación utilizado permite demostrar la efectividad de la metodología para el análisis de estabilidad de los puntos de equilibrio, tanto en forma analítica como en forma gráfica. 
Armonizo con Cruz (2017) en el análisis de resultados de su investigación, es decir, se corrobora el correcto funcionamiento de los modelos implementado para el proceso, en contraste con data de validación de simulaciones desarrolladas en MATLAB/Simulink. El entorno virtual de simulación se desempeña correctamente según las consideraciones esperadas para los distintos escenarios de prueba. Y también con Rodríguez (2013), es decir, el modelo implementado, responde coherentemente a las modificaciones realizadas, la herramienta Simulink de Mtalab brinda la oportunidad de modificar cualquier variable y observar de forma inmediata sus efectos.

Después de obtener los resultados dados por los modelos matemáticos propuestos en la presente investigación, coincido con Ipanaqué et al. (2012) que concluyeron: la vida está gobernada en cierta parte por sistemas de control de nivel, por lo que es inevitable que tenga marcada importancia en el desarrollo industrial. Este campo necesita innovaciones que si no son previamente estudiadas y analizadas podrían quedar inconclusas o simplemente ser inviables e ineficientes. Por esta razón es que se piensa en desarrollar e implementar nuevos sistemas de control, simples y avanzados, en plantas piloto que permitan desarrollar la parte experimental de estas investigaciones. Esto nos permitirá poder llegar a un diseño óptimo que sea verdaderamente útil para la industria. Y finalmente armonizo con Lara et al. (2018) que concluyeron: el trabajo llevado a cabo ha permitido dotar al laboratorio de Control de Procesos de la Universidad de Córdoba de una nueva planta experimental para la enseñanza relacionada con control de nivel, controladores PI, sistemas no lineales y control por ganancia programada.

\section{CONCLUSIONES}

Los modelos matemáticos empleados en la presente investigación, permitieron estimar en menor tiempo con resultados esperados el nivel del agua en un tanque con variaciones generadas por la demanda del servicio, logrando así identificar la estabilidad del sistema.

Las condiciones iniciales consideradas para las variables de entrada del sistema, fueron importantes para linealizar el modelo no lineal y así equilibrar el sistema en menor tiempo con resultados esperados, de estos valores depende el nivel del agua en estado de equilibrio (estabilidad).

Los modelos matemáticos empleados generaron resultados muy similares durante el proceso de simulación del sistema, en la implementación de esta investigación se tomará el modelo lineal, pues este modelo está representado por una función de transferencia expresada en fracciones algebraicas simples 


\section{REFERENCIAS BIBLIOGRÁFICAS}

Albarracín, J. A., \& Argüelles, A. F. (2010). Ingeniería detallada, modelado y simulación de un sistema de tanques interactuantes no lineales [Pontificia Universidad Javeriana]. https://repository.javeriana.edu.co/bitstream/handle/10554/7032/tesis478.pdf?sequence=1\&isAl lowed=y

Anaya, C., Anaya, M. E., \& León, M. F. (2018). Solución numérica y linealizada de un modelo de sistema planar autónomo no lineal, para un equipo de dos tanques interconectados. Pistas Educativas, 40(130). http://www.itc.mx/ojs/index.php/pistas/article/view/1687

Anzurez, J., Padilla, J. A., \& Cuevas, O. (2008). Estabilidad de Sistemas No-lineales: Sistema de Nivel de Líquidos de Dos Tanques Interconectados. RIEE\&C. https://www.itson.mx/publicaciones/rieeyc/Documents/v5/revista_completa_diciembre_2008_v ol5_num2.pdf\#page $=8$

Castaño, S. A. (s. f.). Modelado de sistemas nivel de un tanque [Educativo]. Análisis de sistemas. Recuperado 16 de junio de 2020, de https://controlautomaticoeducacion.com/analisis-desistemas/3-modelado-de-sistemas-nivel-de-un-tanque/

Chapra, S. C., Canale, R. P., Ruiz, R. S. G., Mercado, V. H. I., Díaz, E. M., \& Benites, G. E. (2007). Métodos numéricos para ingenieros (Vol. 5). McGraw-Hill México.

Cruz, W. C. (2017). Desarrollo de un Entorno Virtual para Simulación de un Proceso Hidráulico de 4 Tanques Acoplados [Pontificia Universidad Católica del Perú]. http://tesis.pucp.edu.pe/repositorio/bitstream/handle/20.500.12404/9750/CRUZ_WILLIANS_E NTORNO_VIRTUAL_SIMULACI\%C3\%93N_TANQUES_ACOPLADOS.pdf?sequence=1

Escalante, M. C., \& Villacreses, W. J. (2015). Modelamiento y simulación de un sistema de llenado de dos tanques en serie [B.S. thesis, Espol]. https://www.dspace.espol.edu.ec/bitstream/123456789/30320/1/D-84647.pdf

Fernández, F. A. (2015). Modelado y Simulación de Sistema de Control de Llenado de Estanques mediante Simulink. Escuela Universitaria de Ingeniería Eléctrica y Electrónica, Universidad de Tarapacá, Iquique, Chile.

Gosmann, H. L. (2002). Um sistema multivariável de tanques acoplados para avaliação de técnicas de controle [Universidade de Brasília]. http://www.ene.unb.br/adolfo/Monographs/MScPhD/DM02\%20Hugo\%20Leonardo\%20Gosma nn.pdf

Inga, J. B. (2019). Diseño de un controlador adaptativo neuronal para el control multivariable no lineal del nivel en tres tanques interconectados [Master's Thesis]. Espol. 
Ipanaqué, W., Rodríguez, R., \& Alamo, M. (2012). Modelado y simulación de un sistema de nivel de líquidos, utilizando Simulink® Matlab®. Universidad de Piura.

Lara, M., Garrido, J., Ruz, M. L., \& Vázquez, F. (2018). Control PI adaptativo por ganancia programada del nivel de un tanque de sección trapezoidal. XXXIX Jornadas de Automática, 444-451. https://ruc.udc.es/dspace/bitstream/handle/2183/24915/2018_Lara_Ortiz_Control_PI_adaptativ o.pdf

Loyarte, A. S., \& Clementi, L. A. (2011). Sistema de Control de Niveles con Cuatro Tanques Interconectados: Modelado Matemático y Estimación de Parámetros. XIV Reunión del Procesamiento de la Información y Control (RPIC), Oro Verde, 16-18.

Roa, O. L., Contreras, G. A., Medina, L. V., \& Vega, H. M. (2017). Modelado matemático, simulación, análisis y control de un sistema hidráulico interactivo-tres tanques en serie. Revista de Tecnología, 16(1), 77-98.

Rodríguez, L. A. (2013). Modelo del control de nivel y caudal de líquido en un depósito cilíndrico usando la herramienta Simulink de Matlab. Cap\&Cua, 9(1), 2-7.

Rosa, C. F. (2013). Série de Taylor e aplicações [Universidade Federal de Santa Catarina]. https://repositorio.ufsc.br/bitstream/handle/123456789/105435/Carlos\%20Fabiano\%20Rosa.pdf ?sequence $=1$ 